\title{
Liderazgo escolar y su influencia en el logro de la autonomía de las escuelas chilenas
}

\section{School leadership and its influence on the achievement of autonomy in Chilean schools}

DOI: $10.46932 / \mathrm{sfjdv} 2 \mathrm{n} 2-198$

Received in: March 1st, 2021

Accepted in: May 30th, 2021

\section{Carla Estefanía Olivares Petit}

Doctora (c) en Educación y Magister en didáctica de las ciencias experimentales. Institución actual: Universidad Metropolitana de Ciencias de la Educación - Chile Domicilio completo: Av. José Pedro Alessandri 774, Ñuñoa, Región Metropolitana

Correo electrónico: carla.olivares@umce.cl

\section{Juan Pablo Catalán Cueto}

Doctor en Educación

Institución actual: Universidad Usek Chile

Domicilio completo: Fernando Manterola 0789, Santiago, Providencia, Región Metropolitana Correo electrónico: juanpablo.catalan@usek.cl

\section{RESUMEN}

Como resultado de políticas públicas deficientes que ha presentado Chile a lo largo del tiempo, el gobierno de turno ha emprendido la tarea de implementar una reforma educacional potente caracterizada por la inclusión y educación para todos. Esta reforma se ha plasmado en una nueva ley, que pone fin al lucro en educación, por lo que a partir de 2017 toda institución escolar que reciba subvención del gobierno será considerada del Estado. Por lo anterior, es de suma importancia que todo establecimiento educacional, a partir de su proyecto educativo institucional, logre crear una identidad propia que le otorgue autonomía. El presente artículo hace referencia a la importancia que ejerce un adecuado liderazgo educativo para lograr esa autonomía. Se repasará la literatura con el fin de comprobar la tesis planteada, y se contrastarán las diversas teorías revisadas. Para finalizar, se expondrá un análisis de cómo un líder escolar empoderado puede influenciar en la comunidad educativa para crear una meta común y sentirse identificado con ella, con lo que podrá lograr la deseada autonomía.

Palabras clave: liderazgo escolar, identidad, autonomía institucional, sello educativo, proyecto educativo institucional.

\footnotetext{
ABSTRACT

As a result of deficient public politics that Chile has had a long the time, the government on the run has implemented a powerful Educational Reform that emphasizes on the inclusion and education for everyone. This new law stops the lucrative actions of education, from 2017 on every institution that receives payments from de government will be consider of the State. That said, is very important that every educational establishment have an institutional education project, which will point to the autonomy. The present article, is about the importance that a good leadership has over this autonomy. We pre- 334 Innovación e interculturalidad en la educación sent a look thru the literature so that we can prove our thesis. At the end, there will be an analysis on how a good lider influence the educational community to create a common goal, and feel identify with it, to create the wanted autonomy.
} 
Key words: escolar leadership, identity, institutional autonomy, educational, institutional education Project

\section{INTRODUCCIÓN}

En Chile el liderazgo ha sido un tema que ha adquirido una gran importancia a lo largo de la pasada década. En la actualidad, las distintas reformas han comprendido la importancia de incorporar en sus agendas el liderazgo escolar de los establecimientos educacionales. Asimismo, para ser reconocidos oficialmente como establecimiento educacional, el Ministerio de Educación (de ahora en adelante Mineduc) establece como condición la existencia de un proyecto educativo institucional (de ahora en adelante PEI), el cual organiza, norma, entrega dirección y autonomía a la institución educativa. A través de la contrastación de diversos temas y teorías, este artículo pretende exponer la influencia que ejerce el liderazgo del equipo directivo en lograr la autonomía del establecimiento educacional, para así evidenciar el impacto que genera su adecuado manejo.

Este estudio se realiza en Chile en 2016, en base a revisión de literatura contemporánea, con el fin de acreditar la autonomía que pueda adquirir un establecimiento educacional al verse motivado por un líder que cautiva y enrola a su equipo de trabajo en el PEI. Según lo planteado por Leithwood y Rielh en 2004 (p. 10), el liderazgo hace referencia principalmente a la capacidad de un sujeto para fomentar un compromiso hacia una meta común, y lograr así una responsabilidad mayor por parte de la persona liderada, lo que presume un posterior esfuerzo adicional y una mayor productividad.

A continuación se realizará un recorrido por los distintos conceptos que influyen en lograr la autonomía de un establecimiento educacional, luego se presentará la relación que existe entre autonomía y liderazgo por medio de planteamientos encontrados en la literatura, y se dejará espacio a una mirada reflexiva del tema, para finalmente exponer las conclusiones encontradas en torno a nuestro objetivo y la tesis planteada, así como se detallarán los hallazgos y desafíos propuestos para lograr la mejora.

\section{HACIA UNA DEFINICIÓN CONCRETA}

El liderazgo se puede entender de varias maneras, sin embargo en el presente trabajo haremos referencia al de tipo transformacional, el cual se define como "La capacidad de ejercer influencia sobre otras personas, de manera que éstas puedan tomar las líneas propuestas como premisa para su acción. Esta influencia, no basada en el poder o autoridad formal, se puede ejercer en distintas dimensiones, especialmente en el plano organizativo, cuando una dirección logra alcanzar consenso y moviliza a la organización en torno a metas comunes (Leithwood K. D., 2006)”. 
$\mathrm{Al}$ asociar el liderazgo al ámbito escolar, se desprende el liderazgo educacional, el cual se puede definir como "...la labor de movilizar e influenciar a otros para articular y lograr las intenciones y metas compartidas de la escuela" (Leithwood y Riehl, 2004, p. 20), es decir, toda la comunidad educativa hace suyo el objetivo institucional, y los convoca para un proyecto común de mejora. Por su parte, la autonomía “....es de origen griego y en su composición se identifican dos expresiones 'autos', que significa 'por sí mismo', y 'nomos', que significa ley” (Macri, 2008, p. 1).

Al tomar este concepto y unirlo al ámbito de la educación, Sonia Villarroel (2002, p. 21) plantea el concepto 'autonomía institucional', que define como 'Libertad relativa de los integrantes de una escuela para tomar decisiones, y para definir y proyectar situaciones pedagógicas y curriculares dentro del marco legal vigente. Ejemplos: proyecto educativo institucional, planes y programas de estudio, reglamento de evaluación, entre otros". Asimismo, Mariela Macri en el 2008 (p. 2) sostiene que es posible asumir un autogobierno y una autogestión de la institución, lo que, por su parte, contribuirá a la desconcentración y también a la descentralización. Mencionado lo anterior, podemos evidenciar la importancia de un potente PEI para adquirir la autonomía.

En 2002 Villarroel (p. 29), con apoyo del Mineduc, se refiere al PEI como "Instrumento técnico y político que orienta el quehacer del establecimiento escolar, explicitando su propuesta educacional y especificando los medios que se pondrán en marcha para realizarlo". En ese mismo sentido plantea que “implica el avance en los niveles de participación de todos los actores hacia el logro de una mayor autonomía e identidad para la escuela". Es importante mencionar que en 2015 y con la intención de potenciar la autonomía y lograr una identidad, el Mineduc resalta la relevancia de que la comunidad escolar identifique los sellos educativos, los cuales caracterizan la propuesta educativa de la institución y la hacen singular y distinta al resto de los PEI (p. 28).

\section{LIDERAZGO, PROYECTO EDUCATIVO INSTITUCIONAL Y AUTONOMÍA}

Desde 2006, y con la gran revolución 'pingüina', que movilizó al país, los distintos gobiernos de turno han asumido la gran deuda que se tiene con la educación chilena. Por lo anterior, se han elaborado diversas reformas educacionales con el fin de lograr una educación equitativa y de calidad, y se han establecido políticas públicas que apuntan al derecho a la educación y libertad de enseñanza. Es así como la Ley General de Educación implementada en 2009 establece como principio fundamental a la autonomía y plantea en su artículo 3 que "El sistema se basa en el respeto y fomento de la autonomía de los establecimientos educativos. Consiste en la definición y desarrollo de sus proyectos educativos, en el marco de las leyes que los rijan" (p. 3), al mismo tiempo sostiene en su artículo 10 que "Los sostenedores de establecimientos educacionales tendrán derecho a establecer y ejercer un proyecto educativo, con la 
participación de la comunidad educativa y de acuerdo a la autonomía que le garantice esta ley. También tendrán derecho a establecer planes y programas propios en conformidad a la ley, y a solicitar, cuando corresponda, financiamiento del Estado de conformidad a la legislación vigente (p. 5)”.

Por su parte, la actual Ley de Inclusión, que fomenta la nueva reforma educacional del país, también recoge la importancia de que los establecimientos educacionales posean tal autonomía, la cual impulsa el reconocimiento de la identidad de la institución, y se establece que el PEI "se transformará en el principal referente utilizado por las familias a la hora de elegir un establecimiento educacional, ahora en un sistema con igualdad de oportunidades en el acceso a las unidades educativas financiadas por el Estado" (Mineduc, 2015, p. 8). De esta manera, el Mineduc en 2015 expone que "El Estado reconoce la importancia de la identidad de un colegio o comunidad educativa y su aporte a la pluralidad de la nación" (p. 5).

A pesar de lo anterior, el logro de la autonomía y el reconocimiento de la identidad o el sello educativo de un establecimiento se verán influenciadas por el compromiso y la labor del equipo de trabajo que compone la escuela, liderada, en su mayoría, por el director de la institución, y se resalta que ese liderazgo será posible gracias a la influencia que ejerce el director en temas como la motivación, el desarrollo de habilidades, de prácticas y de las condiciones de trabajo que se disponen para los docentes (Leithwood K. D., 2006).

La Organización para la Cooperación y Desarrollo Económico (OCDE) en 2009 destaca que "el liderazgo escolar es una prioridad de la política educativa en todo el mundo. Una mayor autonomía y una mayor concentración en los resultados educativos y escolares han hecho que sea esencial reconsiderar la función de los líderes escolares" (p. 3).

Por su parte, Stoll y Temperley (2009), citados en "El liderazgo educativo y su papel en la mejora" (Bolívar, 2010, p. 12), sostienen que los lideres escolares pueden influir directamente en el rendimiento de los alumnos, al poseer la autonomía suficiente como para tomar decisiones respecto al currículum, a la selección e incluso a la formación de los docentes observando la estrecha relación entre el liderazgo educativo y el desempeño de los estudiantes. Así, en 2004 Oscar Maureira publica un estudio realizado al liderazgo como factor de la eficacia escolar.

En este se aprecian los efectos directos que puede tener el liderazgo sobre la participación y satisfacción de la comunidad educacional, así como su efecto indirecto sobre la eficacia del centro, con lo que se evidencia el aspecto vital del liderazgo dentro del establecimiento educacional y asociado a variables esenciales para su funcionamiento, como la colaboración y recompensa pedagógica de los profesores (p. 18). 
En estudios realizados por Leithwood y Jantzi (2004, p. 38), se describe el liderazgo transformacional de las escuelas a partir de seis dimensiones: “...crear una visión y metas para la escuela; brindar estimulación intelectual; ofrecer apoyo individualizado; simbolizar las prácticas y los valores profesionales; demostrar expectativas de alto desempeño; y desarrollar estructuras para fomentar la participación en las decisiones de la escuela". Considerando lo anterior, y destacando la importancia del PEI en la adquisición de la autonomía, es relevante mencionar al docente como elemento fundamental de su construcción, como sostiene el Mineduc (2015, p. 9): “ ...es relevante que en el proceso de ajuste, actualización y/o reformulación de su Proyecto Educativo Institucional, participe activamente toda la comunidad educativa liderada por el equipo directivo, el consejo escolar y el sostenedor", pues solo de esta manera lograremos que la meta sea común y compartida por toda la comunidad educativa.

Por otra parte, el Marco de la Buena Dirección (MBD) (2005, p. 11) resalta la importancia del rol del director "en la generación de climas organizacionales adecuados para potenciar el proyecto educativo y los logros de aprendizaje de los estudiantes”. Adicionalmente, Mónica Celis (2010,p. 7) manifiesta que si los docentes desarrollan un sentido de identidad en su institución, con sus correspondientes valores y al mismo tiempo generan un estilo de relación basada en respeto y colaboración, impactará directamente en su desempeño y motivación ante su labor institucional, "variable más influyente en el desempeño de los estudiantes", según lo planteado por la Unesco en 2008 (citado en Celis, 2010, p. 8). Pues como plantea Stephen Anderson (2010, p. 38): "El liderazgo directivo ejerce una influencia indirecta en los aprendizajes de los alumnos, a través de su incidencia en las motivaciones, habilidades y condiciones del trabajo de los profesores, que a su vez afectan los resultados de los estudiantes".

Por su parte, Antonio Bolívar (1997, p. 9) plantea que el director debe ser un líder con una visión clara de los objetivos de su institución, la cual debe lograr compartir y hacer común entre los miembros de la comunidad, y obtener así que todos actúen de acuerdo con ella, y centrar todos los apoyos y recursos en lograr su ejecución. De igual forma, Bolívar (1997, p. 8) sostiene: "Sin una auténtica autonomía institucional es altamente improbable que se llegue a alcanzar en los centros educativos la asunción de valores, metas y normas".

A pesar de lo anterior, Beatriz Pont (2010, p. 5) hizo un estudio respecto al liderazgo escolar ejercido en 22 países pertenecientes a la OCDE y se evidenció que, al considerar todas las exigencias que recaen sobre el director, así como la gran responsabilidad y la carga horaria que poseen, los sueldos no son proporcionales a la cantidad de trabajo se desarrolla, pues como promedio se estima solo un $20 \%$ sobre el sueldo que recibe el profesor.

Como consecuencia existe un bajo interés por ejercer los cargos directivos en los establecimientos. De igual forma, es importante mencionar que el Mineduc (2015) en su “Análisis de indicadores educativos 
de Chile y la OCDE en el contexto de la reforma educacional" hace referencia a los sueldos que reciben los profesores chilenos y resalta: "En 2013, aun cuando el sueldo docente había aumentado durante los últimos años, este no lograba superar el promedio del salario máximo de los países de la OCDE” (p. 19), lo cual apoya la falta de deseo de tener mayor carga laboral al asumir un cargo directivo.

Ríos y Gómez (2013, p. 10) evidencian que a lo largo de los planteamientos hechos por distintos autores acerca de la autonomía que se delega a los establecimientos educacionales en el sur de Europa, se ha intentado dar más responsabilidad y autonomía a la dirección de la escuela, lo que conlleva más responsabilidad de gestión educativa. Sin embargo, ha habido denuncias de los sindicatos de docentes, ya que lo ven como un 'atentado' contra la libertad de enseñanza, contra la libertad pedagógica y contra su cátedra, lo que iría en desmedro de su libertad como profesor. Debido a esto, Barreré (citado en Ríos y Gómez, 2013, p. 13) dice que la autonomía pedagógica de los profesores españoles y franceses de los liceos públicos inhabilita el correcto ejercicio de una dirección pedagógica, y ocasiona un conflicto entre lo que se produce en el aula y en el mismo centro educativo.

Sostienen de esta manera que el liderazgo educativo se puede perjudicar si no se reestructuran las políticas educativas y sus bases, pues no basta con modificar las atribuciones que se entregan al director o a la dirección escolar, si no se trabajan pilares fundamentales de la autonomía, como son el proyecto educativo institucional y un modelo organizativo elaborado en conjunto que facilite el trabajo en equipo, el compromiso y el involucramiento de todos los actores hacia la mejora.

Como bien afirma Samuel Gento (p. 30): "No se trata de poner más poder en manos burocráticas, sino de dotar a los profesionales de los instrumentos necesarios para alcanzar un compromiso para planificar y llevar a cabo iniciativas que mejoren la calidad de la educación...”. Pues, a diferencia de las distintas organizaciones que implementan el liderazgo transformacional como estrategia, en educación se debe enfocar explícitamente en los aprendizajes de los alumnos, ya que el líder (generalmente el director) no remplaza el trabajo docente, más bien lo potencia y cualifica, y se establece así una diferencia en la calidad de la escuela, así como en la educación recibida por los alumnos.

Es importante mencionar que la meta asociada a los aprendizajes hace referencia a dirigir la atención a asegurarse de que todas las acciones y los componentes del sistema escolar apoyen su desarrollo, y no asociado específicamente a la gestión pedagógica en sí. De igual manera se debe considerar que no existe una forma de liderazgo educacional universal, dado que su efectividad dependerá de cada institución y del contexto en el cual se encuentra inmersa (Leithwood y Riehl, 2004, p. 25). 339 Educación superior y su vinculación a la sociedad. 


\section{UNA MIRADA PERSONAL}

Si bien todo establecimiento educacional chileno debe contar, por reglamento, con un proyecto educativo institucional, este no siempre tomará la relevancia que le corresponde, pues ello dependerá de cómo ese proyecto se elabora, y de la gestión del líder y del compromiso que los actores asuman frente a él (docentes, alumnos, apoderados y sostenedor).

Como se puede apreciar a través de la literatura recolectada, el proyecto educativo entregará un sello e identidad al establecimiento educacional, el que plasmará a la institución como única y diferenciada del resto de las instituciones, y le otorgará autonomía en el momento de gestionar acciones curriculares, organizativas, de convivencia, liderazgo y financieras. Por lo anterior, es importante que el PEI sea elaborado con todos los integrantes involucrados en los aprendizajes de los alumnos y la comunidad en general, pues solo así los distintos actores se sentirán parte en la construcción de una visión comunitaria, de la cual son partícipes.

A pesar de lo anterior, dependerá en gran medida del liderazgo que asuma el director de la institución en el momento de plantear y difundir las metas, pues el líder debe ser capaz de impregnar a todo su equipo de trabajo con los objetivos comunes y la identidad escolar. Sin embargo, los líderes no surgen por imposiciones, sino por méritos escasamente cuestionables. Su participación se mide en función de su aporte y reconocimiento moral, no al simple hecho de tener más años o grados, y por ello la tarea no es fácil.

Con esto planteado, cabe la importancia de que el líder se plasme como tal y logre que los actores de la comunidad educativa se sientan parte de esta meta común y la asuman como tal. Con ello se reafirmará el sello educativo, lo que generará así la búsqueda constante por lograr un objetivo compartido, y no así satisfacer solo una meta profesional personal. Lo anterior favorecerá el buen clima laboral, así como el trabajo en equipo, y en consecuencia la colaboración constante en la planificación de estrategias para la mejora escolar, de igual manera en la organización y distribución de recursos con el fin de cumplir con las metas y los objetivos propuestos. Ello reafirmará la identidad del establecimiento, con lo que se concretará y aferrará en mayor medida la idea de una institución con un alto grado de autonomía.

Es importante mencionar la relevancia que la Ley de Inclusión (impulsada por el gobierno de Michelle Bachelet en 2015) entrega a la identidad y autonomía de los establecimientos educacionales, pues será el mayor parámetro con el que contarán los apoderados para elegir el establecimiento educacional en el cual quiere educar a su hijo. Con lo anterior podemos dilucidar cómo esta nueva ley es el impulsador a poner énfasis en el PEI y su autonomía, con lo que se da así mayor aporte al contexto, y con ello a la calidad. 340 Innovación e interculturalidad en la educación. 
Cada familia podrá así optar por la escuela que quiere para su hijo y el tipo de educación que le quiere entregar, lo que generará la idea de comunidad escolar como una familia. Asimismo, la ley permite que al proyecto educativo institucional, el cual debe desarrollar programas de mejoramiento en pro de la calidad, se anexen otros tipos de proyectos, como artes, deportes o exigencia académica, los cuales pueden cumplir con las necesidades específicas que puede estar buscando el apoderado. Pues bien, se sabe que los colegios de mejores resultados en evaluaciones intermedias y en la PSU aplican desde hace décadas modelos educativos que van más allá del aula, y que refuerzan áreas de desarrollo no necesariamente cuantitativamente evaluables.

No obstante, una cosa es lo que plantea el espíritu de la ley, otra lo que las familias quieren. En una sociedad aspiracional como la chilena, donde los padres sostienen el principio de "que los hijos deben ser más que ellos", se busca la calidad, no subvencionada con nombres señoriales, sin que les interese saber de proyectos educativos ni estrategias de enseñanza. Los hijos salen mal preparados y los reciben universidades donde la ilusión del grado académico no les hace evaluar su calidad ni condiciones de enseñanza. En el fondo, nada de lo que los teóricos plantean como lo ideal y justo considera una idiosincrasia como la chilena que, para el caso del sistema escolar en todos sus niveles, solo valida la calificación (la nota) como indicador de calidad, por sobre el aprendizaje y el desarrollo intelectual de los estudiantes.

Un director que ejerza un liderazgo escolar impulsador a propósitos comunes, como es el PEI, generará un mayor involucramiento y un buen clima laboral entre su equipo de trabajo, donde todos apuntan a lograr un objetivo común, lo que fortalecerá alcanzar la autonomía institucional. Con todo lo anteriormente mencionado se puede obtener una buena gestión, elevar las expectativas de los docentes, buenos logros académicos y mayor compromiso de los docentes y apoderados. De igual forma, el establecimiento educacional podrá diferenciarse y distinguirse entre otros de similares características, y se cumplirá así con las necesidades de sus alumnos, de los docentes, así como con el contexto en el cual se encuentra inmerso.

Antes de cualquier cambio, antes de elaborar cualquier propuesta, el sistema educacional debe renovar sus cuadros docentes. Fiarse del liderazgo de quienes dirigen los establecimientos simplemente es desconocer cómo funcionan. En un oficio como el de la docencia escolar, en particular en el sector público, donde existe poca movilidad, sistemas de fiscalización y evaluación prácticamente inexistentes, donde los estímulos son voluntarios y no se toman porque implica un esfuerzo extra, pensar en cambios, autonomías, liderazgos efectivos y mejoras parece ser una utopía.

La historia de la educación en Chile es la de los proyectos inconclusos, básicamente porque la calidad del gremio, por decirlo de modo sutil, nunca ha estado a la altura del enorme desafío que encierra 
formar las futuras generaciones. El tradicional respeto a la figura del docente deriva más del hecho de tener una formación intelectual, que, siendo básica, era muy superior a la del promedio. Eso lo convertía en autoridad. La veneración a los antiguos docentes está también ligado al carácter heroico de la disciplina, a eso de esforzarse mucho a cambio de nada, en una mirada romántica que nunca considera la frustración de los profesores, expresada en un permisivo entorno de violencia física y psicológica.

Hoy ninguno de esos dos escenarios existe, por lo que el oficio es visto con indiferencia por quienes lo ejercen. Te aseguro que en tus años de trabajo siempre has conocido más profesores mediocres que realmente dedicados, con genuina vocación. Si es así, ¿cómo implementar liderazgos? Y, siguiendo la idea, ¿cómo habilitar al director para que lo ejerza?

A modo de síntesis, el liderazgo educacional es un tema complejo, no obstante se evidencia la importancia de un adecuado liderazgo educacional. Tomando en cuenta que el término 'liderazgo' se relaciona con la palabra 'influencia' podemos decir que el líder de un establecimiento educacional será el impulsador o la gran influencia para mover, gestionar y, sobre todo, motivar a su equipo de trabajo en torno a una visión y misión compartida, y establecer consensos respecto a los objetivos más significativos para la institución. Todo lo anterior considerando siempre el contexto educativo en el cual se encuentran inmersos los actores y liderando en torno a las necesidades y cambios que la comunidad presente en pro de la mejora continua y el logro de la autonomía.

\section{CONCLUSIÓN}

A lo largo del presente artículo pudimos dilucidar la gran importancia que tienen el liderazgo educacional, la autonomía y la adecuada implementación del proyecto educativo institucional, y buscar así exponer la relación existente entre un buen liderazgo por parte del equipo directivo y la adquisición de la autonomía institucional.

Como se pudo apreciar, la relación es evidentemente estrecha, ya que un elemento es influyente directo en el otro, por lo que se advierte que sin un líder positivo que impulse la visión del PEI es imposible que el equipo de trabajo se involucre y se sienta parte de él, lo que impide que se refuerce el sello educativo, el cual, por su parte, entrega autonomía y diferencia a la institución del resto, siendo imposible lograr la mejora y calidad que se espera del establecimiento, puesto que no existirán parámetros que orienten o contextualicen al docente en su labor.

Tal como se resalta en la Ley General de Educación, la autonomía se refleja en los proyectos educativos institucionales, que establecen las metas y expectativas, siempre y cuando se haya movilizado a la comunidad educativa a participar en sus logros, lo que origina espacios concretos para involucrarlos y generar redes de participación entre los actores. 
Si bien el MBD no habla específicamente de autonomía, esta se refleja en los distintos principios valóricos que se exponen en el PEI y en los conocimientos profesionales que debe tener el equipo directivo frente a mejoras, cambios a nivel nacional, y que se deben adaptar a su propia realidad y contexto.

Por otro lado, Leithwood sostiene la importancia de que el líder sea impecable en el momento de motivar y cautivar al equipo de trabajo en torno a la visión institucional, y hace un llamado a potenciar constantemente el desarrollo de habilidades, a estimular y resaltar las buenas prácticas docentes, y a estar atento a las condiciones de trabajado que se presentan. Mediante lo recién expuesto podremos cultivar un clima laboral propicio e impulsador para alcanzar metas y objetivos, pues, como sostiene Mónica Celis, un clima estimulante permitirá el trabajo colaborativo y comprometido dentro de la institución. Siguiendo la idea expuesta, la OCDE deja en evidencia la necesidad de implementar en todas las agendas de gobierno la potenciación de líderes escolares con las competencias suficientes como para ser un referente a seguir entre la comunidad educativa, y resaltar a través de un estudio la necesidad de mejorar las condiciones laborales de los profesionales de la educación, así como sus salarios, para que así la función de director sea un puesto de trabajo atractivo y demandado por los profesores.

Por su parte, el gobierno actual se encuentra en vías de implementar la nueva Ley de Inclusión, donde el punto de partida ha sido el fin al lucro, al copago y a la selección. Con ello todos los establecimientos educacionales de Chile pasarán a ser del Estado sin presentar mayor diferenciación el uno del otro. Por ello se hace un llamado para impulsar y mejorar los PEI, para así tener un sello educativo que haga única y distintiva a la institución, y adquirir así mayor autonomía procurando ser impecables en el momento de su constitución.

La autonomía y el liderazgo, ambos desde una perspectiva positiva, permiten actuar de forma razonada y proyectiva, en la que se evalúan situaciones, se emiten juicios sobre lo que consideran eficaz y adecuado para su contexto. Así, de forma indirecta, y según lo expuesto por Anderson, el liderazgo tendrá una poderosa influencia sobre el desempeño y los resultados de los alumnos. Lo anterior obedece al impacto positivo que tiene un liderazgo educativo bien ejercido sobre los distintos actores que componen la comunidad educativa, los cuales, por su parte, se comprometerán con la labor pedagógica y con el sello educativo, con lo que se logrará una autonomía como un pilar arraigado a la cultura propia de la institución.

Es necesario entonces que ante la crisis valórica del último tiempo los directores a quienes se designarán como líderes educativos tengan las aptitudes, los conocimientos correspondientes acordes con los estándares de desempeño para no caer en una autonomía autoritarista ni autoimpuesta. Los encargados de la selección de los próximos líderes directivos deberán centrarse en que estos cumplan con las competencias y los hábitos, que posean una visión estratégica, y que comprendan la importancia del 
trabajo en equipo, que sea empático, con capacidad de escucha, abierto al aprendizaje, flexible y resiliente, entre otros aspectos.

Por su parte, el gobierno de turno deberá centrarse en que el liderazgo sea ejercido como tal, para así eliminar las conductas autoritarias y jerárquicas tan evidenciadas en la sociedad chilena. Se deberán implementar estrategias que permitan percibir si el establecimiento educacional realmente cumple con lo plasmado en el PEI, pues de lo contrario incumpliría con el perfil presentado al apoderado respecto a la institución, siendo pasado a llevar su derecho de elección de dónde educar a su hijo y, peor aún, afectará el proceso de aprendizaje del alumno.

Para finalizar, queda como desafío personal de toda institución educacional dependiente del Estado de Chile reflexionar críticamente, junto con su equipo de trabajo, respecto a sus PEI, analizar las necesidades actuales que puede presentar la comunidad, revisar si realmente responde al contexto en el cual se encuentra inmerso, si posee un sello educativo y si este se identifica con él. Es importante también considerar cuáles serán las acciones a tomar para mejorar, potenciar o cambiar ese proyecto, con la finalidad de que se implemente un liderazgo que permita motivar a los profesionales, y lograr así diferenciarse de otros establecimientos y adquirir mayor autonomía. Es trascendente mencionar que la escuela es el eslabón más pequeño del proceso de la reforma educacional y del proceso de enseñanza, sin embargo es precisamente ahí donde es posible generar el verdadero cambio de la educación. 


\section{REFERENCIAS}

Cornejo, J. (2016). «El café». DED, 2.

Leithwood, K., y Riehl, C. (2004). ¿Cómo liderar nuestras escuelas?: Aportes desde la investigación. Santiago, Chile: Fundación Chile.

Javiera. (2006). Javiera. Javiera, 40.

Ríos, J., y Gómez, E. (2013). Relación entre competencias básicas de los estudiantes y competencias del profesorado. Revista Fuentes, 209-230.

Barrére, A. (2006). «Les chefs d'établissement face aux enseignants: emjeux et conflits de l'autonomie pédagogique». Revue Francaise de Pédagogie.

Gento, S. (1999). «Autonomía del centro educativo, impulsor de la calidad institucional». Acción Pedagógica.

Leithwood, K. D. (2006). Succesful School Leadership. What is and how it Influences Pupil Learning. UK: National College for School Leadership.

Villarroel, S. (2002). Proyecto educativo institucional: Marco legal y estructura básica. Santiago: Mineduc.

Macri, M. (2008). «Descentralización educativa y autonomía institucional: ¿constituye la descentralización un proceso abierto?». Revista Iberoamericana de Educación, 10- 20.

Mineduc (2015). Orientaciones para la revisión y actualización del proyecto educativo institucional. Santiago: Mineduc.

Mineduc (2009). Establece la Ley General de Educación. Santiago: Diario Oficial de la Republica de Chile.

Mineduc (1 de mayo de 2015). Comunidad escolar. Obtenido de www.comunidadescolar.cl OCDE (2009). Mejorar el liderazgo.

OCDE. Bolívar, A. (2010). «El liderazgo educativo y su papel en la mejora: Una revisión actual de sus posibilidades y limitaciones». Psicoperspectiva, 9-33.

Maureira, O. (2004). «El liderazgo, factor de eficacia escolar. Hacia un modelo causal». Revista Electrónica Iberoamericana sobre Calidad, Eficacia y Cambio en Educación.

Mineduc (2005). Marco para la buena dirección. Santiago: Mineduc.

Celis, M. (2010). Gestión de recursos humanos en la escuela. Santiago: Fundación Chile.

Anderson, S. (2010). «Liderazgo directivo: claves para una mejor escuela». Psicoperspectiva, 34-52.

Bolívar, A. (1997). Liderazgo, mejora y centros educativos. Madrid: UNED. 
Ponts, B. (2010). Liderazgo y autonomía del centro escolar. OCDE.

Mineduc (2015). Análisis de indicadores educativos de Chile y la OCDE en el contexto de la reforma educacional: revisión comparada de la educación en Chile en el panorama internacional, en base a la publicación Education at a Glance 2015. Santiago: Mineduc

Eleazar De la Rosa, F., Menacho, I., Alvarez M., Camarena J. (2021). Efectos de estilos de liderazgo del director en la gestión de Instituciones Educativas. Vol. 2 No. 2 (2021): South Florida Journal of Development, Miami, v. 2, n. 2, apr./jun. 2021 